\title{
Partially commutative inverse monoids
}

\author{
Volker Diekert, Markus Lohrey, and Alexander Miller \\ Universität Stuttgart, FMI, Germany \\ diekert, lohrey, miller@informatik.uni-stuttgart.de
}

\begin{abstract}
Free partially commutative inverse monoids are investigated. Analogously to free partially commutative monoids (trace monoids), free partially commutative inverse monoid are the quotients of free inverse monoids modulo a partially defined commutation relation on the generators. An $O(n \log (n))$ algorithm on a RAM for the word problem is presented, and NP-completeness of the generalized word problem and the membership problem for rational sets is shown. Moreover, free partially commutative inverse monoids modulo a finite idempotent presentation are studied. For these monoids, the word problem is decidable if and only if the complement of the commutation relation is transitive.
\end{abstract}

\section{Introduction}

A labelled transition system is deterministic, if in every state there is for each label at most one outgoing edge with this label. Of particular interest are systems where we can perform an undo-operation. This means that the system is codeterministic: For each state and label there is at most one incoming edge with this label. In this setting each label defines a partially defined injective mapping from states to states. The resulting transformations form an inverse monoid; and it is well-known that each inverse monoid arises this way. Because of its close connection to automata theory inverse monoids received quite an attention in theoretical computer science and there is a well-established literature on this subject, see e.g. [11,15].

In this paper we are interested in the situation where the labels describe actions of a (deterministic and codeterministic) transition system and some of the actions can be performed independently. This leads to a partial commutation and therefore to partially commutative inverse monoids. Free partially commutative inverse monoids were first studied in the thesis of da Costa [17], where, among others, the word problem has been shown to be decidable. Da Costa did not prove any complexity bounds. Our first contribution is a new approach to define free partially commutative inverse monoids which is closer to a standard construction of Margolis and Meakin [10]. We use a natural closure operation for subsets of free partially commutative groups (also known as graph groups [5]). Using our construction we are able to show in Section 3 that the word problem of a free partially commutative inverse monoid is solvable in time $O(n \log (n))$ on a RAM. In Section 4, we study the generalized word problem for free partially commutative inverse monoids. The generalized word problem asks whether a given monoid element belongs to a given finitely generated submonoid. In fact, we consider the more general membership problem for rational subsets of a free partially commutative inverse monoid, and we show its NP-completeness. NP-hardness appears already for the 
special case of the generalized word problem for a 2-generator free inverse monoid. It is quite remarkable that the generalized word problem remains decidable in our setting, because it is known to be undecidable for direct products of free groups [13]. So there is an undecidable problem for a direct product of free groups where the same problem is decidable for a direct product of free inverse monoids.

In the second part of the paper we consider free partially commutative inverse monoids modulo a finite idempotent presentations, which is a finite set of identities between idempotent elements. We show that the resulting quotient monoids have decidable word problems if and only if the underlying dependence structure is transitive. In the transitive case, the uniform word problem (where the idempotent presentation is part of the input) turns out to be EXPTIME-complete, whereas for a fixed idempotent presentation the word problem is solvable both in linear time on a RAM and logarithmic space on a Turing machine. These results generalize corresponding results for free inverse monoids modulo an idempotent presentation from $[8,10]$. Our decidability result for the case of a transitive dependence structure is unexpected in light of a result of Meakin and Sapir [12], where it was shown that there exist E-unitary inverse monoids over a finitely generated Abelian group, where the word problem is undecidable. The proof of this result in [12] is quite involved and relies on a sophisticated encoding of computations of Minski machines. In fact, a slight variation of our undecidability proof for non-transitive dependence structures can be used to give a simpler proof for the result of Meakin and Sapir; it will appear in the full version of this paper.

\section{Preliminaries}

In the following let $\Sigma$ be a finite alphabet and $\Sigma^{-1}=\left\{a^{-1} \mid a \in \Sigma\right\}$ be a disjoint copy of $\Sigma$. We let $\Gamma=\Sigma \cup \Sigma^{-1}$. Then $\Gamma$ becomes a set with an involution ${ }^{-1}: \Gamma \rightarrow \Gamma$ by setting $\left(a^{-1}\right)^{-1}=a$ for all $a \in \Sigma$. We extend this involution to an involution ${ }^{-1}: \Gamma^{*} \rightarrow \Gamma^{*}$ by setting $\left(a_{1} \cdots a_{n}\right)^{-1}=a_{n}^{-1} \cdots a_{1}^{-1}$ for $a_{i} \in \Gamma, 1 \leq i \leq n, n \geq 0$. The free group generated by $\Sigma$ is denoted by $F(\Sigma)$; it can be defined as the quotient monoid $\Gamma^{*} /\left\{a a^{-1}=1 \mid a \in \Gamma\right\}$.

Let $\mathcal{M}$ be a finitely generated monoid and let $\Gamma$ be a finite generating set for $\mathcal{M}$, i.e., there exists a surjective homomorphism $h: \Gamma^{*} \rightarrow \mathcal{M}$. The word problem for $\mathcal{M}$ is the computational problem that asks for two given words $u, v \in \Gamma^{*}$, whether $h(u)=h(v)$. The generalized word problem for $\mathcal{M}$ asks whether for given words $u, v_{1}, \ldots, v_{n}$ the element $h(u)$ belongs to the submonoid $\left\{h\left(v_{1}\right), \ldots, h\left(v_{n}\right)\right\}^{*} \subseteq \mathcal{M}$ generated by $h\left(v_{1}\right), \ldots, h\left(v_{n}\right)$. It is easy to see that the decidability/complexity of the (generalized) word problem does not depend on the underlying set of generators. Now let $\mathcal{G}$ be a finitely generated group and let $\Gamma$ be a finite generating set for $\mathcal{G}$. The Cayley graph of $\mathcal{G}$ w.r.t. $\Gamma$ is the undirected graph $\mathcal{C}(\mathcal{G}, \Gamma)=\left(\mathcal{G},\left\{\{u, v\} \mid u^{-1} v \in \Gamma\right\}\right)$. Note that the undirected edge $\{u, v\}$ can be viewed as a pair of directed edges $(u, v)$, labelled with $u^{-1} v \in \Gamma$, and $(v, u)$, labelled with $v^{-1} u \in \Gamma$.

\subsection{Free partially commutative inverse monoids}

A monoid $\mathcal{M}$ is called inverse if for every $x \in \mathcal{M}$ there is a unique $x^{-1} \in \mathcal{M}$ such that

$$
x x^{-1} x=x \text { and } \quad x^{-1} x x^{-1}=x^{-1} .
$$


It is well-known that uniqueness of the inverse $x^{-1}$ follows, if we require additionally to (1) that for all $x, y \in \mathcal{M}$ :

$$
x x^{-1} y y^{-1}=y y^{-1} x x^{-1}
$$

Elements $x x^{-1}$ are exactly the idempotents in $\mathcal{M}$. It is clear that every mapping $\varphi$ : $\Sigma \rightarrow \mathcal{M}$ to an inverse monoid $\mathcal{M}$ lifts uniquely to a homomorphism $\varphi: \Gamma^{*} \rightarrow \mathcal{M}$ such that $\varphi\left(u^{-1}\right)=\varphi(u)^{-1}$ for all $u \in \Gamma^{*}$. By an independence relation we mean here an irreflexive and symmetric relation $I_{\Gamma} \subseteq \Gamma \times \Gamma$ such that $(a, b) \in I_{\Gamma}$ implies $\left(a^{-1}, b\right) \in I_{\Gamma}$ for all $a, b \in \Gamma$. Note that $I$ is specified by $I_{\Sigma}=I_{\Gamma} \cap \Sigma \times \Sigma$, and we may view $\left(\Sigma, I_{\Sigma}\right)$ as an undirected graph. For words $u, v \in \Gamma^{*}$ we write $(u, v) \in I_{\Gamma}$ if $u=a_{1} \cdots a_{m}, v=b_{1} \cdots b_{n}$, and $\left(a_{i}, b_{j}\right) \in I_{\Gamma}$ for all $i \leq m, j \leq n$.

Every inverse monoid $\mathcal{M}$ can be viewed as a monoid of partially defined injections over a set $Q$. Thus, if $a \in \mathcal{M}$, then $a$ is an injection $a: \operatorname{dom}(a) \hookrightarrow Q$ where $\operatorname{dom}(a) \subseteq$ $Q$. Now partially defined injections $a$ and $b$ can be called independent, if the following three conditions are satisfied for all $q \in Q$ :

(i) if $q \in \operatorname{dom}(a)$, then: $a(q) \in \operatorname{dom}(b) \Longleftrightarrow q \in \operatorname{dom}(b)$,

(ii) if $q \in \operatorname{dom}(b)$, then: $b(q) \in \operatorname{dom}(a) \Longleftrightarrow q \in \operatorname{dom}(a)$,

(iii) if $q \in \operatorname{dom}(a) \cap \operatorname{dom}(b)$, then: $a b(q)=b a(q)$.

These conditions look technical, but a brief reflection shows that they are indeed natural translations of an intuitive meaning of independence. Note that (i)-(iii) is a stronger requirement than to say that $a$ and $b$ commute. Consider the following situations:
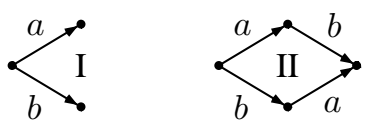

In situation I the transitions $a$ and $b$ are not independent although they commute: The result of $a b$ is the same as $b a$; it is the undefined mapping, which corresponds to a zero in the monoid. It is also clear that $a$ and $b$ should not be called independent in this situation because $a$ can disable $b$ (and vice versa). The situation II is different: The set $Q$ has four states; it corresponds to the set of global states of the asynchronous product of two independent components. The first (second, resp.) component can only perform the action $a(b$, resp.). A simple calculation shows that if we define independence in $\mathcal{M}$ as above by (i)-(iii), then the independence of $a$ and $b$ implies the independence of $a^{-1}$ and $b$, too. This motivates the following definition: An inverse monoid over $\left(\Sigma, I_{\Sigma}\right)$ is an inverse monoid $\mathcal{M}$ together with a mapping $\varphi: \Sigma \rightarrow \mathcal{M}$ such that $\varphi(a) \varphi(b)=\varphi(b) \varphi(a)$ and $\varphi(a)^{-1} \varphi(b)=\varphi(b) \varphi(a)^{-1}$ for all $(a, b) \in I_{\Sigma}$. Thus, we can define the free inverse monoid over $\left(\Sigma, I_{\Sigma}\right)$ by

$$
\operatorname{FIM}(\Sigma, I)=\operatorname{FIM}(\Sigma) /\left\{a b=b a, a^{-1} b=b a^{-1} \mid(a, b) \in I_{\Sigma}\right\} .
$$

Here, $\operatorname{FIM}(\Sigma)$ denotes the free inverse monoid over $\Sigma$, which is defined as the quotient monoid of $\Gamma^{*}=\left(\Sigma \cup \Sigma^{-1}\right)^{*}$ modulo the equations in (1) and (2) for all $x, y \in$ $\Gamma^{*}$. In the following, we will briefly write $I$ for both $I_{\Sigma}$ and $I_{\Gamma}$. It will be always clear, on which set $I$ is defined. The monoid $\operatorname{FIM}(\Sigma, I)$ is also called a free partially commutative inverse monoid. 
Da Costa has studied $\operatorname{FIM}(\Sigma, I)$ in his thesis from a more general viewpoint of graph products [17]. As a consequence he showed that $\operatorname{FIM}(\Sigma, I)$ has a decidable word problem. In his construction he used the general approach via Schützenberger graphs and Stephen's iterative procedure [16]. The decidability of the word problem follows because da Costa can show that Stephen's procedure terminates. However, no complexity bounds are given in [17].

Another starting point for defining free partially commutative inverse monoids is a construction of Margolis and Meakin [9]. One would start with the free partially commutative group $G(\Sigma, I)$ (defined below) and consider as elements of an inverse monoid the pairs $(A, g)$, where $A$ is a finite and connected subgraph of the Cayley graph of $G(\Sigma, I)$ with $1, g \in A$. Although this construction yields for $I=\emptyset$ indeed $\operatorname{FIM}(\Sigma)$ by a result of Munn [14], it fails for $I \neq \emptyset$, simply because independent generators do not commute. Thus, we have to do something else. Fortunately it is enough to modify the construction of Margolis and Meakin slightly in order to achieve a simple and convenient description of the elements in $\operatorname{FIM}(\Sigma, I)$. Our approach is based on the notion of coherently prefix-closed subsets which we make precise in the next section.

\subsection{Trace monoids and graph groups}

Recall that $\Gamma=\Sigma \cup \Sigma^{-1}$ and $I \subseteq \Gamma \times \Gamma$ is an irreflexive and symmetric relation such that $(a, b) \in I$ implies $\left(a^{-1}, b\right) \in I$. Let $M(\Gamma, I)=\Gamma^{*} /\{a b=b a \mid(a, b) \in I\}$ be the free partially commutative monoid (or trace monoid) over $(\Gamma, I)$. Due to $(a, b) \in I \Rightarrow$ $\left(a^{-1}, b\right) \in I$, the involution ${ }^{-1}: \Gamma^{*} \rightarrow \Gamma^{*}$ is well-defined on $M(\Gamma, I)$. The relation $D=(\Gamma \times \Gamma) \backslash I$ is called the dependence relation.

There is a rich theory on trace monoids [4]. Here we need some basic results, only. The perhaps most important fact ist that traces (i.e., elements of $M(\Gamma, I)$ ) have a unique description as dependence graphs, which are node-labelled acyclic graphs. Let $u=$ $a_{1} \cdots a_{n} \in \Gamma^{*}$ be a word. The vertex set of the dependence graph of $u$ is $\{1, \ldots, n\}$ and vertex $i$ is labelled with $a_{i} \in \Gamma$. There is an edge from vertex $i$ to $j$ if and only if $i<j$ and $\left(a_{i}, a_{j}\right) \in D$. Now, two words define the same trace in $M(\Gamma, I)$ if and only if their dependence graphs are isomorphic.

A clique covering of the dependence relation $D$ is a tuple $\left(\Gamma_{i}\right)_{1 \leq i \leq k}$ such that $\Gamma=$ $\bigcup_{i=1}^{k} \Gamma_{i}$ and $D=\bigcup_{i=1}^{k} \Gamma_{i} \times \Gamma_{i}$. W.l.o.g. we may assume that $a \in \Gamma_{i}$ if and only if $a^{-1} \in \Gamma_{i}$. Let $\pi_{i}: M(\Gamma, I) \rightarrow \Gamma_{i}^{*}$ the projection homomorphism which deletes all letters from $\Gamma \backslash \Gamma_{i}$. The morphism $\pi: M(\Gamma, I) \rightarrow \prod_{i=1}^{k} \Gamma_{i}^{*}$ defined by $\pi(u)=$ $\left(\pi_{1}(u), \ldots, \pi_{k}(u)\right)$ is injective [2,3]. For $u, v \in M(\Gamma, I)$ we write $u \leq v$ if $u$ is a prefix of $v$, i.e., $v=u w$ in $M(\Gamma, I)$ for some trace $w$. A trace $f$ is a factor of $u$, if we can write $u=p f q$ in $M(\Gamma, I)$. Let $\max (u)=\{a \in \Gamma \mid u=t a$ for some trace $t\}$; it is the set of labels of the maximal nodes in the dependence graph of $u$.

An important fact about traces is the following: Assume we have $u \leq w$ and $v \leq w$ for some $u, v, w \in M(\Gamma, I)$. Then the supremum $u \sqcup v \in M(\Gamma, I)$ w.r.t. the prefix order $\leq$ exists. We can define $u \sqcup v$ by restricting the dependence graph of $w$ to the domain of $u$ and $v$, where $u$ and $v$ are viewed as downward-closed subsets of the dependence graph of $w$. If $\left(\Gamma_{i}\right)_{1 \leq i \leq k}$ is a clique covering of the dependence relation, then for every $i$, either $\pi_{i}(u) \leq \pi_{i}(v)$ and $\pi_{i}(u \sqcup v)=\pi_{i}(v)$ or $\pi_{i}(v) \leq \pi_{i}(u)$ and $\pi_{i}(u \sqcup v)=\pi_{i}(u)$. A trace $p$ is a prime if $|\max (p)|=1$. 
For $t \in M(\Gamma, I)$ let $\mathbb{P}(t)=\{p \leq t \mid p$ is a prime $\}$. Note that $t=\sqcup \mathbb{P}(t)$ (the supremum of the traces in $\mathbb{P}(t))$. Let $A \subseteq M(\Gamma, I)$. We define $\mathbb{P}(A)=\bigcup_{t \in A} \mathbb{P}(t)$. The set $A$ is called prefix-closed, if $u \leq v \in A$ implies $u \in A$. It is called coherentlyclosed if for every $C \subseteq A$ such that $\sqcup C$ exists, $\sqcup C \in A$. One can show that $A$ is coherently-closed if for all $u, v \in A$ such that $u \sqcup v$ exists, $u \sqcup v \in A$. In the following we say that $A$ is closed, if it is both prefix-closed and coherently-closed. Clearly, for every $A \subseteq M(\Gamma, I)$ there is a smallest closed set $\bar{A}$ with $A \subseteq \bar{A}$. One has $\bar{A}=$ $\{\sqcup C \mid C$ is a set of prefixes of $A, \sqcup C$ exists $\}$ and $\bar{A}=\overline{\bar{A}}$. The notions of a prime and coherence are standard in domain theory and the connection to trace theory is exposed in [4, Sec. 11.3].

Lemma 1. For $A, B \subseteq M(\Gamma, I)$ we have $\bar{A}=\bar{B}$ if and only if $\mathbb{P}(A)=\mathbb{P}(B)$.

A trace rewriting system $R$ over $M(\Gamma, I)$ is just a finite subset of $M(\Gamma, I) \times M(\Gamma, I)$ [3]. We can define the one-step rewrite relation $\rightarrow_{R} \subseteq M(\Gamma, I) \times M(\Gamma, I)$ by: $x \rightarrow_{R} y$ if and only if there are $u, v \in M(\Gamma, I)$ and $(\ell, r) \in R$ such that $x=u \ell v$ and $y=u r v$. The notion of a confluent and terminating trace rewriting system is defined as for other types of rewriting systems. A trace $u$ is an irreducible normal form of $t$ if $t \stackrel{*}{\rightarrow}_{R} u$ and there does not exist a trace $v$ with $u \rightarrow_{R} v$.

The free partially commutative group (or graph group [5]) over $(\Sigma, I)$, briefly $G(\Sigma, I)$, is the quotient of the free group $F(\Sigma)$ modulo the defining relations $a b=b a$ for all $(a, b) \in I$. Clearly, $G(\Sigma, I)=M(\Gamma, I) /\left\{a a^{-1}=1 \mid a \in \Gamma\right\}$. We can define a confluent and terminating trace rewriting system $R=\left\{a a^{-1} \rightarrow 1 \mid a \in \Gamma\right\}$, where $1 \in M(\Gamma, I)$ denotes the empty trace. Given $u \in \Gamma^{*}$ we can view $u \in M(\Gamma, I)$ and compute its irreducible normal form $\widehat{u} \in M(\Gamma, I)$ w.r.t. $R$ in linear time [3]. Thus, $\widehat{u}$ is a trace without any factor of the form $a a^{-1}$ for $a \in \Gamma$. We also say that the trace $\widehat{u}$ is reduced. We have $u=v$ in $G(\Sigma, I)$ if and only if $\widehat{u}=\widehat{v}$. This allows to solve the word problem in $G(\Sigma, I)$ in linear time $[3,19]$. In the following, whenever $u \in \Gamma^{*}$ (or $u \in M(\Gamma, I)$ or $u \in G(\Sigma, I))$, then $\widehat{u} \in M(\Gamma, I)$ denotes this unique reduced trace such that $u=\widehat{u}$ in $G(\Sigma, I)$. The set $\widehat{M}(\Gamma, I)=\{\widehat{u} \mid u \in M(\Gamma, I)\}$ is in canonical oneto-one correspondence with $G(\Sigma, I)$, hence we may identify $\widehat{u}$ with the group element it represents.

A subset $A \subseteq G(\Sigma, I)$ is called closed, if the set of reduced traces $\widehat{A}=\{\widehat{g} \in$ $\widehat{M}(\Gamma, I) \mid g \in A\}$ is closed. Clearly, for every $A \subseteq G(\Sigma, I)$, there is a smallest closed subset $\bar{A} \subseteq G(\Sigma, I)$ such that $A \subseteq \bar{A}$. We have $\bar{A}=\overline{\bar{A}}$ and we can identify $\bar{A}$ with $\overline{\widehat{A}} \subseteq \widehat{M}(\Gamma, I)$. Note that $\widehat{M}(\Gamma, I)$ is closed. Recall that $I$ is irreflexive, hence $\left\{1, a, a^{-1}\right\}$ is closed since $\left(a, a^{-1}\right) \in D$. We now give a geometric interpretation of closed sets. Let $g, h \in G(\Sigma, I)$. A geodesic between $g$ and $h$ is a shortest path in the Cayley graph of $G(\Sigma, I)$. The labelling of such a path is unique as a reduced trace $\widehat{u} \in \widehat{M}(\Gamma, I)$ such that $g \widehat{u}=h$ in $G(\Sigma, I)$. We say that $f \in G(\Sigma, I)$ is on a geodesic from $g$ to $h$ if $\widehat{f} \leq \widehat{u}$.

Proposition 1. A subset $A \subseteq G(\Sigma, I)$ is closed if and only if both $1 \in A$ and whenever $f$ is on a geodesic from $g$ to $h$ with $g, h \in A$, then $g f \in A$, too.

Corollary 1. Let $A \subseteq G(\Sigma, I)$ be closed and $g \in A$. Then $g^{-1} A$ is closed, too. 
Proof. Since $g \in A$, we have $1 \in g^{-1} A$. The property " $f$ is on a geodesic from $h_{1}$ to $h_{2}$ with $h_{1}, h_{2} \in A$ implies $h_{1} f \in A$ " is invariant by translation. Thus $A$ satisfies this property if and only if $g^{-1} A$ satisfies this property.

\subsection{A realization of free partially commutative inverse monoids}

We are now ready to give a concrete realization of the free inverse monoid over $(\Sigma, I)$. The realization is very much in the spirit of Margolis and Meakin [9], but differs in the subtle point that we allow closed subsets of $G(\Sigma, I)$, only. Consider the set of pairs $(A, g)$ where $A \subseteq G(\Sigma, I)$ is a finite and closed subset of the graph group $G(\Sigma, I)$ and $g \in A$. This set becomes a monoid by

$$
(A, g) \cdot(B, h)=(\overline{A \cup g B}, g h) .
$$

An immediate calculation shows that the operation is associative and that $(\{1\}, 1)$ is a neutral element. Moreover, the idempotents are the elements of the form $(A, 1)$ and idempotents commute. By Corollary 1, if $g \in A \subseteq G(\Sigma, I)$ and $A$ is closed, then $g^{-1} A$ is closed, too. Hence we can define $(A, g)^{-1}=\left(g^{-1} A, g^{-1}\right)$. A simple calculation shows that (1) and (2) are satisfied. Thus our monoid is an inverse monoid. We view $\Gamma$ as a subset of this monoid by identifying $a \in \Gamma$ with the pair $(\{1, a\}, a)$, and this yields a canonical homomorphism $\gamma$ defined by $\gamma(u)=(\overline{\{\widehat{v} \mid v \leq u\}}, \widehat{u})$ for $u \in \Gamma^{*}$. We obtain $\gamma(a b)=(\{1, a\}, a) \cdot(\{1, b\}, b)=(\overline{\{1, a, a b\}}, a b)$. Now, if $(a, b) \in I$, then $\overline{\{1, a, a b\}}=\{1, a, b, a b\}=\overline{\{1, b, b a\}}$, i.e., $\gamma(a b)=\gamma(b a)$. Hence, we obtain an inverse monoid over $(\Sigma, I)$ since $(a, b) \in I$ implies $\left(a^{-1}, b\right) \in I$. As a consequence, the homomorphism $\gamma$ can be viewed as a canonical homomorphism

$$
\gamma: \operatorname{FIM}(\Sigma, I) \rightarrow\{(A, g) \mid g \in A \subseteq G(\Sigma, I), A \text { finite and closed }\} .
$$

Theorem 1. The morphism $\gamma$ in (3) is an isomorphism.

Proof. Consider a pair $(A, g)$ with $A \subseteq G(\Sigma, I)$ finite and closed and $g \in A$. Recall that $\widehat{A}=\{\widehat{u} \in \widehat{M}(\Gamma, I) \mid u \in A\}$. Let $w \in \Gamma^{*}$ be an arbitrary word representing the trace $\left(\prod_{\widehat{u} \in \widehat{A}} \widehat{u} \widehat{u}^{-1}\right) \widehat{g}$ where the product is taken in any order. Then a simple reflection shows $\gamma(w)=(A, g)$. Hence $\gamma$ is surjective. It remains to show that $\gamma$ is injective. To see this let $w \in \Gamma^{*}$ and $\gamma(w)=(A, g)$. Note that $\widehat{w}=\widehat{g}$. It suffices to show

$$
w=\left(\prod_{u \in \widehat{A}} u u^{-1}\right) \widehat{w} \text { in } \operatorname{FIM}(\Sigma, I)
$$

This is enough because it implies $\gamma(w)=\gamma\left(w^{\prime}\right) \Longrightarrow w=w^{\prime}$ in $\operatorname{FIM}(\Sigma, I)$ for all $w, w^{\prime} \in \Gamma^{*}$. If $w=\varepsilon$ then $(A, g)=(\{1\}, 1)$ and (4) is true. Hence let $w=v a$ with $a \in \Gamma$. By induction $v=\left(\prod_{u \in \widehat{B}} u u^{-1}\right) \widehat{v}$ in $\operatorname{FIM}(\Sigma, I)$, where $\gamma(v)=(B, h)$. We obtain $\widehat{A}=\widehat{\widehat{B} \cup\{\widehat{w}\}}$ and $w=\left(\prod_{u \in \widehat{B}} u u^{-1}\right) \widehat{v} a$ in $\operatorname{FIM}(\Sigma, I)$.

We distinguish whether $\widehat{v} a$ is reduced or not. If $\widehat{v} a$ is not reduced, then $\widehat{v}=\widehat{w} a^{-1} \in$ $\widehat{B}$, i.e., $\widehat{w} \in \widehat{B}$ since $\widehat{B}$ is prefix-closed. It follows $\widehat{B}=\widehat{A}$. We obtain in $\operatorname{FIM}(\Sigma, I)$ :

$$
w=\left(\prod_{u \in \widehat{B}} u u^{-1}\right) \widehat{v} a=\left(\prod_{u \in \widehat{A}} u u^{-1}\right) \widehat{w} a^{-1} a=\left(\prod_{u \in \widehat{A}} u u^{-1}\right) \widehat{w} \widehat{w}^{-1} \widehat{w} a^{-1} a
$$




$$
=\left(\prod_{u \in \widehat{A}} u u^{-1}\right) \widehat{w} a^{-1} a \widehat{w}^{-1} \widehat{w}=\left(\prod_{u \in \widehat{A}} u u^{-1}\right) \widehat{v} \widehat{v}^{-1} \widehat{w}=\left(\prod_{u \in \widehat{A}} u u^{-1}\right) \widehat{w} .
$$

It remains the case where $\widehat{v a}=\widehat{v} a=\widehat{w}$. We obtain in $\operatorname{FIM}(\Sigma, I)$ :

$$
w=\left(\prod_{u \in \widehat{B}} u u^{-1}\right) \widehat{w}=\left(\prod_{u \in \widehat{B}} u u^{-1}\right) \widehat{w} \widehat{w}^{-1} \widehat{w} .
$$

Clearly, $\widehat{w} \in \widehat{A}$. Hence $w=\left(\prod_{u \in A^{\prime}} u u^{-1}\right) \widehat{w}$ in $\operatorname{FIM}(\Sigma, I)$ for some subset $A^{\prime} \subseteq \widehat{A}$ such that $\overline{A^{\prime}}=\widehat{A}$ (set $A^{\prime}=\widehat{B} \cup\{\widehat{w}\}$ ). Therefore it is enough to show $\prod_{u \in A^{\prime}} u u^{-1}=$ $\prod_{u \in \widehat{A}} u u^{-1}$ in $\operatorname{FIM}(\Sigma, I)$. This is the assertion of the following claim.

Claim: Let $A \subseteq M(\Gamma, I)$. Then $\prod_{u \in A} u u^{-1}=\prod_{u \in \bar{A}} u u^{-1}$ in $\operatorname{FIM}(\Sigma, I)$.

To prove this claim, let $v \leq u \in A$. Then $u=v w=v v^{-1} v w=v v^{-1} u$ in $\operatorname{FIM}(\Sigma, I)$. Hence we may assume that $A$ is prefix closed. Now, let $u, v \in A$ such that $w=u \sqcup v$ exists. Then, by Levi's lemma (see e.g. [4, p. 10]), $u=p r, v=p s$, and $w=$ $p s r$ with $(r, s) \in I$. We obtain in $\operatorname{FIM}(\Sigma, I): u u^{-1} v v^{-1}=p r r^{-1} p^{-1} p s s^{-1} p^{-1}=$ $p r r^{-1} s s^{-1} p^{-1}=p r s s^{-1} r^{-1} p^{-1}=w w^{-1}$. This means that we may assume that $A$ is coherently-closed, too. But if $A$ is both prefix-closed and coherently-closed, then $A=\bar{A}$ by definition of $\bar{A}$. Hence the claim and the theorem follow.

For $I=\emptyset$, Theorem 1 yields Munn's theorem [14] as a special case. Note that for $I=\emptyset$, the closure $\bar{A}$ of a prefix-closed subset $A$ of the free group $F(\Sigma)$ equals $A$ itself. It follows that $\gamma(u)=(\{\widehat{v} \mid v \leq u\}, \widehat{u})$, where $\widehat{v} \in \Gamma^{*}$ is the unique irreducible word corresponding to $v \in \Gamma^{*}$. The set $\{\widehat{v} \mid u \leq v\}$ is also called the Munn tree of $u$.

Since we are interested in computational problems, we are concerned with the input size of elements in $\operatorname{FIM}(\Sigma, I)$. The standard representation is just a word $u$ over the alphabet $\Gamma$. If $\gamma(u)=(A, g)$, then $|A| \leq|u|^{k}$, where $k$ is the number of cliques in a clique covering for the dependence relation, because $t \in \widehat{A}$ implies that $\pi_{i}(t)$ is a prefix of $\pi_{i}(u)$ for all $1 \leq i \leq k$. Hence, for a fixed $(\Sigma, I)$, the size of $A$ is bounded polynomially in the length of $u$, and moreover $A$ can be calculated in polynomial time from $u$. Thus, for computational problems in or above polynomial time, we can represent $(A, g) \in \operatorname{FIM}(\Sigma, I)$ by listing all the elements of $A$ followed by $g$. In fact, instead of writing down the closed set $A$, it suffices to list the primes in $\mathbb{P}(A)$ by Lemma 1. The set $\mathbb{P}(A)$ has size at most $|u|$ whatever $(\Sigma, I)$ is. But in general, the more concise representation is still the standard representation.

\section{The word problem in $\operatorname{FIM}(\Sigma, I)$}

Using Munn's theorem [14], it is easy to solve the word problem for a free inverse monoid in linear time on a RAM. For free partially commutative inverse monoids the solution of the word problem is more involved. We are able to present an $O(n \log (n))$ algorithm on a RAM by using a sophisticated combination of simple data structures:

Theorem 2. For a fixed free partially commutative inverse monoid $\operatorname{FIM}(\Sigma, I)$, the word problem can be solved in time $O(n \log (n))$ on a RAM. 
Proof. Let $u, v \in \Gamma^{*}$. By [3,19], we can test in linear time whether $u=v$ in $G(\Sigma, I)$. It remains to check equality of the closures. Let $\left(\Gamma_{i}\right)_{1 \leq i \leq k}$ be a clique covering of the dependence relation $D=(\Gamma \times \Gamma) \backslash I$. With $w \in \Gamma^{*}$ we associate the following data:

- the prefix-closed set of words $T_{i}(w)=\left\{\pi_{i}(\widehat{s}) \mid s \leq w\right\}$,

- the word $p_{i}(w)=\pi_{i}(\widehat{w}) \in T_{i}$ for every $1 \leq i \leq k$,

- the set of primes $P(w)=\mathbb{P}(\{\widehat{s} \mid s \leq w\})$

- the linearly ordered (w.r.t. the prefix order) set $P_{i}(w)=\mathbb{P}(\widehat{w}) \cap\left\{p \mid \max (p) \in \Gamma_{i}\right\}$.

By Lemma 1, we have to check whether $P(u)=P(v)$. Before we present an efficient implementation of the data structures above, let us first show how to compute $\left(T_{i}(w a), p_{i}(w a), P_{i}(w a)\right)_{1 \leq i \leq k}, P(w a)$ from $\left(T_{i}(w), p_{i}(w), P_{i}(w)\right)_{1 \leq i \leq k}, P(w)$ for $a \in \Gamma$. For this, we have to distinguish the two cases $a^{-1} \notin \max (\widehat{w})$ and $a^{-1} \in$ $\max (\widehat{w})$. We use the following notation: For $a \in \Gamma$ such that $a$ occurs in $t$ define the prime $\delta_{a}(t)$ as the maximal prefix of $t$ such that $\max \left(\delta_{a}(t)\right)=\{a\}$. In case that $a$ does not occur in $t$ let $\delta_{a}(t)=1$. We obtain $\delta_{a}(t a)=\left(\sqcup\left\{\delta_{b}(t) \mid(a, b) \in D\right\}\right) a$.

Case 1. $a^{-1} \notin \max (\widehat{w})$, i.e., $\widehat{w a}=\widehat{w} a$. We have:

$$
\begin{aligned}
& T_{i}(w a)=\left\{\begin{array}{ll}
T_{i}(w) \cup\left\{p_{i}(w) a\right\} & \text { if } a \in \Gamma_{i} \\
T_{i}(w) & \text { otherwise }
\end{array} \quad p_{i}(w a)= \begin{cases}p_{i}(w) a & \text { if } a \in \Gamma_{i} \\
p_{i}(w) & \text { otherwise }\end{cases} \right. \\
& P_{i}(w a)= \begin{cases}P_{i}(w) \cup\left\{\delta_{a}(\widehat{w} a)\right\} & \text { if } a \in \Gamma_{i} \\
P_{i}(w) & \text { otherwise }\end{cases}
\end{aligned}
$$

Note that

$$
\delta_{a}(\widehat{w} a)=\left(\sqcup\left\{\delta_{b}(\widehat{w}) \mid(a, b) \in D\right\}\right) a=\left(\sqcup\left\{\max P_{i}(w) \mid 1 \leq i \leq k, a \in \Gamma_{i}\right\}\right) a .
$$

Case 2. $a^{-1} \in \max (\widehat{w})$, i.e., $\widehat{w}=s a^{-1}$ and $\widehat{w a}=s$ for an irreducible trace $s \in$ $\widehat{M}(\Gamma, I)$. We have $\max P_{i}(w)=\delta_{a^{-1}}(\widehat{w})$ for all $i$ with $a^{-1} \in \Gamma_{i}$ (i.e., $a \in \Gamma_{i}$ ) and:

$$
\begin{array}{ll}
T_{i}(w a)=T_{i}(w) & p_{i}(w a)= \begin{cases}v & \text { if } a^{-1} \in \Gamma_{i}, p_{i}(w)=v a^{-1} \\
p_{i}(w) & \text { otherwise }\end{cases} \\
P(w a)=P(w) \cup \mathbb{P}(s)=P(w) & P_{i}(w a)= \begin{cases}P_{i}(w) \backslash \max P_{i}(w) & \text { if } a^{-1} \in \Gamma_{i} \\
P_{i}(w) & \text { otherwise }\end{cases}
\end{array}
$$

For the equality $P(w) \cup \mathbb{P}(s)=P(w)$ note that $\mathbb{P}(s) \subseteq P(w)$ since the trace $s$ is a prefix of the trace $\widehat{w}$. Let us now discuss an efficient implementation of our data structures such that the updates above can be done in time $O(\log (n))$. The prefix-closed set $T_{i}(w)$ can be stored as a trie with at most $\left|\pi_{i}(w)\right|$ many nodes, i.e., a rooted tree, where every node has for every $a \in \Gamma_{i}$ at most one $a$-labelled outgoing edge and $T_{i}(w)$ equals the set of all path-labels from the root to tree nodes. We assign with every node of $T_{i}(w)$ a key from $\mathbb{N}$. The root gets the key 1 , and with every new node of $T_{i}(w)$ the key is increased by one. This allows to calculate $\max U$ for a subset $U \subseteq T_{i}(w)$, which is linearly ordered by the prefix relation, in time $O(|U|)$ by comparing the keys 
for the nodes in $U$. The word $p_{i}(w)=\pi_{i}(\widehat{w})$ is just a distinguished node of the trie $T_{i}(w)$. Clearly, $a^{-1} \in \max (\widehat{w})$ if and only if $p_{i}(w)$ ends with $a^{-1}$ for all $1 \leq i \leq k$ with $a^{-1} \in \Gamma_{i}$. This means that whenever $a^{-1} \in \Gamma_{i}$, then $p_{i}(w)$ is the $a^{-1}$-successor of its parent node. This allows to distinguish between case 1 and case 2 above in time $O(k)=O(1)$. In case 1 , we have to add an $a$-successor to the node $p_{i}(w)$ in case $a \in \Gamma_{i}$ and $p_{i}(w)$ does not have an $a$-successor yet. This new node becomes $p_{i}(w a)$. If $a \in \Gamma_{i}$ but $p_{i}(w)$ already has an $a$-successor $v$, then $v$ becomes $p_{i}(w a)$. In case 2 , the tries do not change, but if $a \in \Gamma_{i}$, then $p_{i}(w a)$ is the father node of $p_{i}(w)$.

The set of primes $P(w)$ is stored as the set of tuples $\left\{\left(\pi_{i}(t)\right)_{1 \leq i \leq k} \mid t \in P(w)\right\}$, where every projection $\pi_{i}(t)$ is represented by the corresponding node in the trie $T_{i}(w)$. For the set $P(w)$ we use a data structure, which allows $O(\log (n))$ time implementations for the operations insert and find. The linearly ordered set $P_{i}(w)$ is stored as a list of tuples $\left(\pi_{i}(t)\right)_{1 \leq i \leq k}$ for $t \in P_{i}(w)$. Using this representation, the necessary updates for case 2 are clearly possible in constant time. For case 1 , we have to calculate the tuple corresponding to $\delta_{a}(\widehat{w} a)=\left(\sqcup\left\{\max P_{i}(w) \mid 1 \leq i \leq k, a \in \Gamma_{i}\right\}\right) a$. Note that

$$
\pi_{j}\left(\delta_{a}(\widehat{w} a)\right)=\sqcup\left\{\pi_{j}\left(\max P_{i}(w)\right) \mid 1 \leq i \leq k, a \in \Gamma_{i}\right\} \pi_{j}(a)
$$

for $1 \leq j \leq k$. The $\sqcup$ in (5) refers to the prefix order on words. Note that since $\max P_{i}(w)$ is a prefix of the trace $\widehat{w}$ for every $i$, the set $\left\{\pi_{j}\left(\max P_{i}(w)\right) \mid 1 \leq i \leq\right.$ $\left.k, a \in \Gamma_{i}\right\}$ is linearly ordered by the prefix relation on $\Gamma_{j}^{*}$, i.e., the supremum exists. Moreover, this supremum can be computed in time $O(k)$, where $k$ is the number of cliques (which is a constant) by using the keys associated with the nodes from $T_{j}(w)$. This concludes the description of our data structures. Now for our input words $u, v \in$ $\Gamma^{*}$ we first compute $\left(T_{i}(u), p_{i}(u), P_{i}(u)\right)_{1 \leq i \leq k}, P(u)$ and $\left(T_{i}(v), p_{i}(v), P_{i}(v)\right)_{1 \leq i \leq k}$, $P(v)$ in time $O(n \log (n))$. When building up the tries $T_{i}(u)$ and $T_{i}(v)$ we have to use the same node name for a certain string over $\Gamma_{i}$. Then we can check $P(u)=P(v)$ in time $O(n \log (n))$ using the set data structures for $P(u)$ and $P(v)$.

For the uniform word problem, where the independence relation $I$ is part of the input, the above algorithm still yields a polynomial time algorithm. More precisely, the running time is $O\left(\left(k^{2}+\log (n)\right) n\right)$, where $k$ is the number of cliques in a clique covering for the dependence relation and $n$ is the length of the input words.

\section{The generalized word problem in $\operatorname{FIM}(\Sigma, I)$}

In this section, we show that the generalized word problem for a free partially commutative inverse monoid is NP-complete in general. An NP upper bound can be even shown for the membership problem in rational sets of $\operatorname{FIM}(\Sigma, I)$. A rational subset of $\operatorname{FIM}(\Sigma, I)$ is represented concisely by a finite automaton over the alphabet $\Gamma$.

Theorem 3. For every fixed free partially commutative inverse monoid $\operatorname{FIM}(\Sigma, I)$, the membership problem for rational subsets of $\operatorname{FIM}(\Sigma, I)$ belongs to NP.

Proof. For given $(B, g) \in \operatorname{FIM}(\Sigma, I)$ and a finite automaton $\mathcal{A}$ over the alphabet $\Gamma$ we have to determine whether $(B, g) \in \gamma(L(\mathcal{A}))$, where $\gamma: \Gamma^{*} \rightarrow \operatorname{FIM}(\Sigma, I)$ is the 
canonical morphism. In the following, we view $B$ as a subgraph of the Cayley graph of $G(\Sigma, I)$. In a first step we guess a connected subset $C \subseteq B$ with $1, g \in C$ such that its closure $\bar{C}$ equals $B$. It remains to check in NP whether there is a path from 1 to $g$ in $C$, which visits all nodes of $C$ and such that this path is labelled with a word from $L(\mathcal{A})$.

Let $n$ be the number of states of the automaton $\mathcal{A}$. Assume that $p=\left(v_{1}, \ldots, v_{m}\right)$ is a path in $C$ such that $v_{1}=1, v_{m}=g, C=\left\{v_{1}, \ldots, v_{m}\right\}$ and let $q_{1} \ldots, q_{m}$ be a corresponding path in the automaton $\mathcal{A}$, where $q_{1}$ is the initial state and $q_{m}$ is a final state. Let $i_{1}<\cdots<i_{\ell}$ be exactly those positions $j \in\{2, \ldots, m\}$ such that $v_{j} \notin\left\{v_{1}, \ldots, v_{j-1}\right\}$. Clearly, $\ell<|C|$. Set $i_{0}=1$ and $i_{\ell+1}=m+1$. Assume that $\left|i_{k+1}-i_{k}\right|>|C| \cdot n$ for some $k \in\{0, \ldots, \ell\}$. Then there are positions $i_{k} \leq \alpha<\beta<$ $i_{k+1}$ such that $v_{\alpha}=v_{\beta}$ and $q_{\alpha}=q_{\beta}$. It follows that $v_{1}, \ldots, v_{\alpha}, v_{\beta+1}, \ldots, v_{m}$ is a again a path in $C$ from 1 to $g$, which visits all nodes of $C$, and $q_{1}, \ldots, q_{\alpha}, q_{\beta+1}, \ldots, q_{m}$ is a corresponding path in the automaton $\mathcal{A}$.

From the above consideration it follows that if there exists a path from 1 to $g$ in $C$, which visits all nodes of $C$ and such that this path is labelled with a word from $L(\mathcal{A})$, then there exists such a path of length at most $|C|^{2} \cdot n$. Such a path can be guessed in polynomial time. This finishes the proof.

NP-hardness can be already shown for the generalized word problem of $\operatorname{FIM}(\{a, b\})$ :

Theorem 4. The generalized word problem for $\operatorname{FIM}(\{a, b\})$ is NP-hard.

Proof. We prove the theorem by a reduction from SAT. Let $\Psi=\left\{C_{1}, \ldots, C_{m}\right\}$ be a set of clauses over variables $x_{1}, \ldots, x_{n}$. Let $k=m+n$. For $1 \leq i \leq n$ let $P_{i}=\left\{j \mid x_{i} \in\right.$ $\left.C_{j}\right\}$ and $N_{i}=\left\{j \mid \neg x_{i} \in C_{j}\right\}$. Let $u=\left(A, a^{n}\right) \in \operatorname{FIM}(\{a, b\})$, where (the subgraph of the Cayley graph of $F(\{a, b\})$ induced by) $A$ looks as follows:

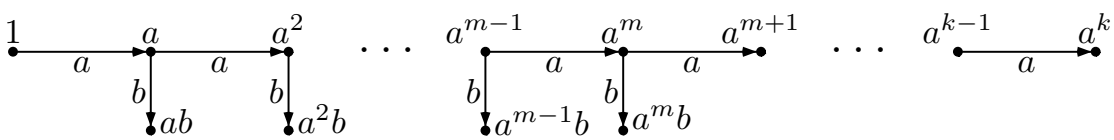

The idea is that the node $a^{j} b$ represents the clause $C_{j}$. For every $1 \leq i \leq n$ define $u_{i, t}=\left(A_{i, t}, a\right) \in \operatorname{FIM}(\{a, b\})$ and $u_{i, f}=\left(A_{i, f}, a\right) \in \operatorname{FIM}(\{a, b\})$, where:

$$
\begin{aligned}
& A_{i, t}=a^{-i+1}\left(\left\{1, a, \ldots, a^{k}\right\} \cup\left\{a^{j} b \mid j \in P_{i}\right\}\right) \subseteq F(\{a, b\}) \\
& A_{i, f}=a^{-i+1}\left(\left\{1, a, \ldots, a^{k}\right\} \cup\left\{a^{j} b \mid j \in N_{i}\right\}\right) \subseteq F(\{a, b\})
\end{aligned}
$$

We claim that $u \in\left\{u_{1, t}, u_{1, f}, \ldots, u_{n, t}, u_{n, f}\right\}^{*}$ if and only if $\Psi$ is satisfiable. First assume that $\Psi$ is satisfied and let $\sigma:\left\{x_{1}, \ldots, x_{n}\right\} \rightarrow\{$ true, false $\}$ be a satisfying assignment. Let $u_{i}=u_{i, t}$ if $\sigma\left(x_{i}\right)=$ true, otherwise set $u_{i}=u_{i, f}$. Then we have $u=$ $u_{1} \cdots u_{n}$, which shows $u \in\left\{u_{1, t}, u_{1, f}, \ldots, u_{n, t}, u_{n, f}\right\}^{*}$. For the other direction assume that $u=u_{1} \cdots u_{m}$, where $m \geq 0$ and $u_{1}, \ldots, u_{m} \in\left\{u_{1, t}, u_{1, f}, \ldots, u_{n, t}, u_{n, f}\right\}$. Since $u=\left(A, a^{n}\right)$ and every $u_{i}$ is of the form $(B, a)$ for some $B$, we have $m=n$. Moreover, since $u_{1} \cdots u_{i-1}$ is of the form $\left(C, a^{i-1}\right)$ for $1 \leq i \leq n$, we must have $u_{i} \in\left\{u_{i, f}, u_{i, t}\right\}$, because otherwise we would obtain $a^{-1} \in A$ (if $u_{i} \in\left\{u_{j, f}, u_{j, t}\right\}$ for $j>i$ ) or $a^{k+1} \in A$ (if $u_{i} \in\left\{u_{j, f}, u_{j, t}\right\}$ for $j<i$ ). Now we can define a truth assignment $\sigma:\left\{x_{1}, \ldots, x_{n}\right\} \rightarrow\{$ true, false $\}$ as follows: $\sigma\left(x_{i}\right)=$ true if $u_{i}=u_{i, t}$ and $\sigma\left(x_{i}\right)=$ false if $u_{i}=u_{i, f}$. Since $a^{j} b \in A$ for every $1 \leq j \leq m$, it follows that for 
every $1 \leq j \leq m$ there is an $1 \leq i \leq n$ such that either $j \in P_{i}$ and $u_{i}=u_{i, t}$ (i.e., $\sigma\left(x_{i}\right)=$ true) or $j \in N_{i}$ and $u_{i}=u_{i, f}$ (i.e., $\sigma\left(x_{i}\right)=$ false). Thus, $\sigma$ satisfies $\Psi$.

The NP upper bound in Theorem 3 generalizes to the uniform case, where the independence relation $I$ is part of the input but the number of cliques in a clique covering for $D=I \backslash(\Sigma \times \Sigma)$ is fixed by a constant. If we give up this restriction, the complexity goes up to PSPACE-completeness:

Theorem 5. The following problem is PSPACE-complete:

INPUT: An independence relation $I \subseteq \Sigma \times \Sigma$ and words $u, u_{1}, \ldots, u_{n} \in \Gamma^{*}$ QUESTION: $u \in\left\{u_{1}, \ldots, u_{n}\right\}^{*}$ in $\operatorname{FIM}(\Sigma, I)$ ?

\section{$5 \operatorname{FIM}(\Sigma, I)$ modulo an idempotent presentation}

Let $I \subseteq \Sigma \times \Sigma$ be an independence relation. An idempotent presentation over $(\Sigma, I)$ is a finite set of identities $P=\left\{\left(e_{i}, f_{i}\right) \mid 1 \leq i \leq n\right\}$, where every $e_{i}$ and $f_{i}$ is an idempotent element in $\operatorname{FIM}(\Sigma, I)$. Based on a reduction to Rabin's tree theorem, Margolis and Meakin have shown that for $I=\emptyset$, the uniform word problem for quotient monoids of the form $\operatorname{FIM}(\Sigma) / P$ (with $P$ idempotent) is decidable [10]. Here, "uniform" means that the idempotent presentation $P$ is part of the input. Recently, in [8] it was shown that the uniform word problem is EXPTIME-complete and that for every fixed idempotent presentation $P$ the word problem for $\operatorname{FIM}(\Sigma) / P$ can be solved in logspace.

In this section we prove that the uniform word problem for monoids of the form $\operatorname{FIM}(\Sigma, I) / P$ (with $P$ an idempotent presentation over $(\Sigma, I)$ ) is decidable if and only if the dependence relation $D=(\Sigma \times \Sigma) \backslash I$ is transitive, and for the transitive case we prove EXPTIME-completeness. Clearly, EXPTIME-hardness follows directly from [8]. For the upper bound, we use analogously to [10] a closure operation on subsets of $G(\Sigma, I)$. Assume that $P$ is an idempotent presentation. Consider a pair $(e, f) \in P$. Then we have $e=(E, 1)$ and $f=(F, 1)$, where $E$ and $F$ are finite and closed subsets of the graph group $G(\Sigma, I)$ and $1 \in E \cap F$. In the following, we identify the pair $(E, 1)$ with the finite closed set $E$. Since $e$ and $f$ are idempotents of $\operatorname{FIM}(\Sigma, I)$, we can replace the relation $e=f$ by the two relations $e=e f$ and $f=e f$ without changing the quotient monoid [10]. Hence, for every pair $(E, F) \in P$, we can assume $E \subseteq F$.

Now assume that $A, B \subseteq G(\Sigma, I)$ are finite and closed. We write $A \Rightarrow_{P} B$ if and only if there exists $(E, F) \in P$ (hence $E \subseteq F)$ and $f \in G(\Sigma, I)$ such that $f E \subseteq A$ and $B=\overline{A \cup f F}$. It is easy to see that the relation $\Rightarrow_{P}$ is strongly confluent, i.e., if $A \Rightarrow_{P} B$ and $A \Rightarrow_{P} C$, then there exists $D$ such that $B \Rightarrow_{P} D$ and $C \Rightarrow_{P} D$. Hence, $A \stackrel{*}{\Leftrightarrow} \stackrel{\text { If }}{P}_{P} B$ if and only if there exists $C$ such that $A \stackrel{*}{\Rightarrow}_{P} C$ and $B \stackrel{*}{\Rightarrow}_{P} C$. Define $\operatorname{cl}_{P}(A)=\bigcup\left\{B \subseteq G(\Sigma, I) \mid A \stackrel{*}{\Rightarrow}_{P} B\right\} \subseteq G(\Sigma, P)$.

Lemma 2. Let $(A, g),(B, h) \in \operatorname{FIM}(\Sigma, I)$. Then $(A, g)=(B, h)$ in $\operatorname{FIM}(\Sigma, I) / P$ if and only if $g=h$ in $G(\Sigma, I)$ and $\operatorname{cl}_{P}(A)=\operatorname{cl}_{P}(B)$.

Using Lemma 2, we can generalize the EXPTIME upper bound from [8] for the case $I=\emptyset$ : If $I \subseteq \Sigma \times \Sigma$ is an independence relation with $D=(\Sigma \times \Sigma) \backslash I$ transitive, then $\Sigma$ is a disjoint union of $D$-cliques $\Sigma_{1}, \ldots, \Sigma_{n}$. Hence, $\operatorname{FIM}(\Sigma, I)$ is the direct product $\prod_{i=1}^{n} \operatorname{FIM}\left(\Sigma_{i}\right)$ of free inverse monoids. Moreover, a closed set $A \subseteq G(\Sigma, I)$ is a direct 
product $A=\prod_{i=1}^{n} A_{i}$ with $A_{i} \subseteq F\left(\Sigma_{i}\right)$ closed. By embedding each of the free groups $F\left(\Sigma_{i}\right)$ into a free group $F(\Theta)$ for a sufficiently large alphabet $\Theta$, we can represent $A$ by an $n$-tuple $\left(A_{1}, \ldots, A_{n}\right)$ of closed subsets $A_{i} \subseteq F(\Theta)$. Computing the closure $\operatorname{cl}_{P}(A)$ corresponds to computing the simultaneous fixpoint of a monotonic mapping on $F(\Theta)^{n}$. Analogously to [8], we can formalize this fixpoint computation in the modal $\mu$-calculus, interpreted over the Cayley graph of $F(\Theta)$. But for this, we need in contrast to [8] the modal $\mu$-calculus with simultaneous fixpoints. On the other hand, the latter logic can be translated into the "ordinary" modal $\mu$-calculus [1] without increasing the complexity of the model-checking problem. Since the model-checking problem of the modal $\mu$-calculus over context-free graphs (which include Cayley graphs of free groups) belongs to EXPTIME [7, 18], we finally get:

Theorem 6. The following problem is EXPTIME-complete:

INPUT: An independence relation $I \subseteq \Sigma \times \Sigma$ with $(\Sigma \times \Sigma) \backslash I$ transitive, an idempotent presentation $P$ over $(\Sigma, I)$ and words $u, v \in \Gamma^{*}$.

QUESTION: $u=v$ in $\operatorname{FIM}(\Sigma, I) / P$ ?

For a fixed idempotent presentation, we can again generalize a corresponding result from [8]:

Theorem 7. If $I \subseteq \Sigma \times \Sigma$ is an independence relation with $(\Sigma \times \Sigma) \backslash I$ transitive and $P$ is an idempotent presentation over $(\Sigma, I)$, then the word problem for $\operatorname{FIM}(\Sigma, I) / P$ can be solved in (i) linear time on a RAM and (ii) logspace on a Turing machine.

Theorem 6 and 7 are an interesting contrast to a result from [12] stating that the variety of E-unitary inverse monoids over an Abelian cover has an undecidable word problem. The difference is that in our setting pairs we only consider pairs $(A, g)$, where $A$ has to be closed, whereas in [12] this restriction is not imposed.

For a non-transitive dependence relation $D$ we can encode the acceptance problem for a Turing-machine $T$ in the word problem for $\operatorname{FIM}(\Sigma, I) / P$. Let $\Sigma=\{a, b, c\}$ and assume that $(a, c),(b, c) \in D$ but $(a, b) \in I$. Then $a$ and $b$ generate in the Cayley graph of $G(\{a, b, c\}, I)$ a two dimensional grid. Using the letter $c$, which is dependent from both $a$ and $b$, we can encode a labelling of the grid-points with tape symbols and states of $T$. With the rewrite relation $\Rightarrow_{P}$ we generate a labelling consistent with the transition function of $T$ and the input of $T$. Hence, we have:

Theorem 8. Let $I \subseteq \Sigma \times \Sigma$ be an independence relation with $D=(\Sigma \times \Sigma) \backslash I$ not transitive. Then there exists an idempotent presentation $P$ over $(\Sigma, I)$ such that the word problem for $\operatorname{FIM}(\Sigma, I) / P$ is undecidable.

For the generalized word problem of $\operatorname{FIM}(\Sigma, I) / P$, we can prove undecidability even for a transitive dependence relation: Let $\Sigma=\{a, b, c, d\}, I=\{a, b\} \times\{c, d\} \cup\{c, d\} \times$ $\{a, b\}$, and let the idempotent presentation $P$ contain all identities $\alpha \alpha^{-1}=1$ for $\alpha \in \Gamma$. Then $\operatorname{FIM}(\Sigma, I) / P$ is a direct product of two free groups of rank 2. By [13], this group has an undecidable generalized word problem. The only remaining case is a dependence relation, which consists of one clique of arbitrary size together with isolated nodes. The corresponding free partially commutative group is of the form $F \times \mathbb{Z}^{k}$, where $F$ is a 
free group of arbitrary rank. It remains open, whether for such a dependence relation, the generalized word problem for $\operatorname{FIM}(\Sigma, I) / P$ is decidable for every idempotent presentation $P$. For the group $F \times \mathbb{Z}^{k}$ the generalized word problem is decidable [6].

\section{References}

1. H. Bekic. Definable operation in general algebras, and the theory of automata and flowcharts. In Programming Languages and Their Definition, LNCS 177, pages 30-55. Springer, 1984.

2. R. Cori and D. Perrin. Automates et commutations partielles. RAIRO - Inform. Théor. Appl., 19:21-32, 1985.

3. V. Diekert. Combinatorics on Traces. LNCS 454. Springer, 1990.

4. V. Diekert and G. Rozenberg, editors. The Book of Traces. World Scientific, 1995.

5. C. Droms. Graph groups, coherence and three-manifolds. J. Algebra, 106(2):484-489, 1985.

6. I. Kapovich, R. Weidmann, and A. Myasnikov. Foldings, graphs of groups and the membership problem. Internat. J. Algebra Comput., 15(1):95-128, 2005.

7. O. Kupferman and M. Y. Vardi. An automata-theoretic approach to reasoning about infinitestate systems. In Proc. CAV 2000, LNCS 1855, pages 36-52. Springer, 2000.

8. M. Lohrey and N. Ondrusch. Inverse monoids: decidability and complexity of algebraic questions. submitted, short version appeared in Proc. MFCS 2005, LNCS 3618, pages 664 675. Springer, 2005.

9. S. Margolis and J. Meakin. E-unitary inverse monoids and the Cayley graph of a group presentation. J. Pure Appl. Algebra, 58(1):45-76, 1989.

10. S. Margolis and J. Meakin. Inverse monoids, trees, and context-free languages. Trans. Amer. Math. Soc., 335(1):259-276, 1993.

11. S. Margolis, J. Meakin, and M. Sapir. Algorithmic problems in groups, semigroups and inverse semigroups. In Semigroups, Formal Languages and Groups, pages 147-214. Kluwer, 1995.

12. J. Meakin and M. Sapir. The word problem in the variety of inverse semigroups with Abelian covers. J. London Math. Soc. (2), 53(1):79-98, 1996.

13. K. A. Mihailova. The occurrence problem for direct products of groups. Math. USSR Sbornik, 70:241-251, 1966. English translation.

14. W. Munn. Free inverse semigroups. Proc. London Math. Soc., 30:385-404, 1974.

15. M. Petrich. Inverse semigroups. Wiley, 1984.

16. J. Stephen. Presentations of inverse monoids. J. Pure Appl. Algebra, 63:81-112, 1990.

17. A. Veloso da Costa. $\Gamma$-Produtos de Monóides e Semigrupos. PhD thesis, Universidade do Porto, 2003.

18. I. Walukiewicz. Pushdown processes: games and model-checking. Inform. and Comput., 164(2):234-263, 2001.

19. C. Wrathall. The word problem for free partially commutative groups. J. Symbolic Comput., 6(1):99-104, 1988. 